\title{
CINÉTICA DA SECAGEM DA SEMENTE DE Moringa oleifera LAM
}

\author{
F. N. C. ALMEIDA ${ }^{1 *}$, J. V. BERNI ${ }^{1}$, J. F. MEDEIROS ${ }^{1}$, N. W. SIQUEIRA ${ }^{1}$, N. C. PEREIRA ${ }^{1}$ \\ ${ }^{1}$ Universidade Estadual de Maringá, Departamento de Engenharia Química \\ *e-mail: fnc.almeida@gmail.com
}

\begin{abstract}
RESUMO
O estudo da cinética de secagem é de fundamental importância para a modelagem matemática, a qual tem sido muito usada no projeto e em análise dos processos de transferência de calor e de massa, durante a secagem. O objetivo do presente trabalho foi ajustar diversos modelos matemáticos ao processo de secagem das sementes de Moringa oleifera Lam. em diferentes temperaturas de secagem, e determinar o coeficiente de difusão efetivo e a energia de ativação. As sementes de moringa utilizadas foram colhidas com teor de água médio de 7,0\% (b.s.). A secagem do produto foi realizada nas temperaturas de $40,50,60,70$ e $80^{\circ} \mathrm{C}$ e velocidade do ar constante de $1,5 \mathrm{~m} / \mathrm{s}$. Dentre os modelos que apresentaram melhor ajuste aos dados experimentais, $\mathrm{o}$ da Aproximação da Difusão e de Verna foram os que melhor se ajustaram aos dados nas diferentes condições de secagem, com exceção para secagem a $40^{\circ} \mathrm{C}$, que apresentou $\mathrm{o}$ modelo Logarítimico como o mais adequado. O coeficiente de difusão efetivo aumentou com a elevação da temperatura, variando de $0,79 \times 10^{-10}$ a $2,14 \times 10^{-10} \mathrm{~m}^{2} / \mathrm{s}$, para $\mathrm{o}$ intervalo de temperaturas de 40 a $80^{\circ} \mathrm{C}$. A relação entre o coeficiente de difusão efetivo e a temperatura de secagem pode ser descrita pela equação de Arrhenius, que apresenta uma energia de ativação para a difusão líquida na secagem de $24,33 \mathrm{~kJ} / \mathrm{mol}$.
\end{abstract}

\section{INTRODUÇÃO}

A Moringa oleifera Lamarck é uma das 14 espécies pertencentes ao único gênero da família Moringacea. Planta arbórea, que pode alcançar de 5 a $10 \mathrm{~m}$ de altura, gerando flores e frutos em um ano. Apresenta sementes globulares, com cerca de $1 \mathrm{~cm}$ de diâmetro e envoltas por uma parede de epiderme grossa, conforme a Figura 1 (MORTON, 1991).

A planta é típica de trópicos úmidos ou terras quentes e secas, adaptam-se bem as condições precárias do solo e são pouco afetadas pelas secas. A moringa é forte, desenvolve-se rapidamente e não requer cuidados especiais (MORTON, 1991). No
Brasil, é encontrada, predominantemente, na região nordeste.

Figura 1 - Semente de moringa com e sem casca.

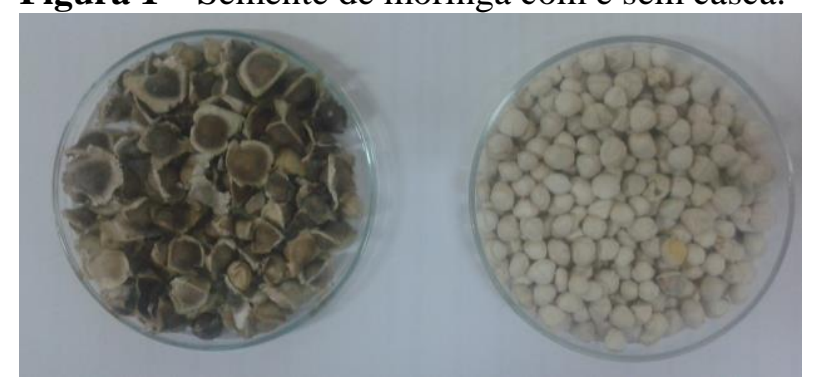

Fonte: Autor (2015).

A árvore de moringa possui importância para a indústria, medicina, agricultura e como alimento. Sua semente é utilizada nos processos de tratamento de água e efluentes, 
devido à presença de uma proteína floculante, que age como coagulante natural (FRIGHETTO et al., 2007).

Segundo Anwar e Bhanger (2003), a semente é composta em $40 \%$ do seu peso por óleo, com predominância do ácido oleico (76\%). O óleo extraído da semente, conhecido comercialmente como óleo "Ben" ou "Behen", apresenta alto valor alimentício e industrial, sendo utilizado no preparo de alimentos, na fabricação de cosméticos, lubrificantes e combustíveis.

$\mathrm{O}$ estudo do processo de secagem fornece informações relativas ao comportamento do fenômeno de transferência de calor e massa entre o material biológico e o elemento de secagem, normalmente ar atmosférico, aquecido ou não; essas informações são fundamentais para o projeto, operação e simulação de sistemas de secagem e secadores (AFONSO JÚNIOR e CORRÊA, 1999)

No caso de sementes, a secagem é aplicada para reduzir o teor de umidade de produtos agrícolas, a fim de preservar a qualidade das sementes. Também é responsável por provocar uma redução do peso e do volume substancial do material, minimizando gastos com transporte $\mathrm{e}$ armazenamento (DOYMAZ, 2005).

Além disso, a secagem é usualmente operada antes do processo de extração do óleo, pois aumenta o contato entre o solvente e o soluto (óleo), resultando em maiores teores e menor custo de produção.

Estudos sobre sistemas de secagem, seu dimensionamento, otimização e a determinação da viabilidade de sua aplicação comercial, podem ser feitos por simulação matemática. A simulação busca um modelo matemático satisfatório, para representar a perda de umidade do produto durante $\mathrm{o}$ período de secagem (AFONSO JÚNIOR e CORRÊA, 1999).

O estudo e a modelagem matemática da cinética de secagem têm despertado o interesse de vários pesquisadores para os mais diferentes produtos. Entretanto, há poucos estudos relatados sobre a secagem da semente de moringa.

Por este motivo, o presente trabalho tem como objetivo estudar a cinética da secagem em camada fina das sementes de moringa submetidas a diferentes temperaturas de secagem, ajustando modelos matemáticos aos dados experimentais. E também, determinar o coeficiente de difusividade e a energia de ativação.

\section{MATERIAIS E MÉTODOS}

As sementes de Moringa oleifera foram cultivadas, na Fazenda Experimental de Iguatemi (FEI), campus da Universidade Estadual de Maringá (UEM). A colheita foi realizada manualmente, no período de abril a junho de 2014, apresentando teor de água em torno de $7,2 \%$ (b.s.) e espessura média de 6,8 $\mathrm{mm}$. Em seguida, as sementes com cascas foram armazenadas em sacos plásticos, em refrigeradores $\mathrm{a}-15^{\circ} \mathrm{C}$.

Antes de cada experimento, foram retiradas do freezer e mantidas em temperatura ambiente por pelo menos 1 hora, para então serem descascadas manualmente. A Figura $1 \mathrm{O}$ teor de umidade, em base seca, foi determinado antes de iniciar cada experimento por meio do método de secagem direta em estufa a $105^{\circ} \mathrm{C}$ por $24 \mathrm{~h}$, conforme a Equação 1

$\mathrm{X}(\mathrm{b}$. s. $)=\frac{\mathrm{M}_{\text {úmida }}-\mathrm{M}_{\text {seca }}}{\mathrm{M}_{\text {seca }}}$

A secagem das sementes de moringa foi realizada em um secador convectivo de bancada com fluxo transversal, de acordo com a Figura 2, localizado no Laboratório de Engenharia Química II do DEQ/UEM.

O módulo possui um soprador de ar com ajuste de velocidade, ligado a um duto metálico equipado com quatro resistências elétricas, responsáveis por aquecerem o ar. $\mathrm{O}$ 
ar aquecido segue até uma bandeja perfurada, que serve de suporte para a amostra. A indicação da temperatura do ar é feita por um controlador de temperatura (Robertshaw Modelo LDT-800) inserido logo abaixo da bandeja perfurada do secador. A velocidade do ar é monitorada por anemômetro digital portátil (Instrutherm - Modelo TAVR-650).

Figura 2 - Módulo experimental de secagem, sistema convectivo.

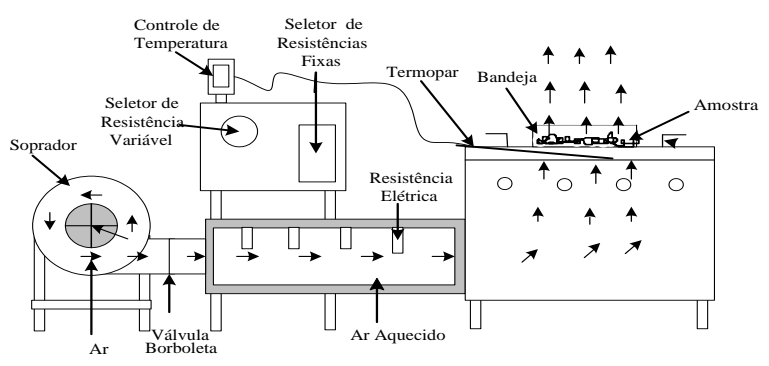

Fonte: Luz, 2006.

A determinação das curvas de secagem (umidade versus tempo) da semente de moringa foi realizada, em duplicata, nas temperaturas de $40,50,60,70$ e $80{ }^{\circ} \mathrm{C}$. A variação da velocidade do ar foi mantida constante em $1,5 \mathrm{~m} / \mathrm{s}$.

$\mathrm{O}$ experimento de secagem foi monitorado por gravimetria, pesando-se o conjunto bandeja-amostra em intervalos regulares. Em cada temperatura, a massa da peneira com a amostra foi pesada, inicialmente, a cada 2 minutos em balança digital (GEHAKA - Modelo Bg 4000 precisão: $0,01 \mathrm{~g}$ ) e a partir de 80 minutos, o intervalo de tempo de pesagem passou a ser a cada 4 minutos. Este processo foi realizado até que três pesagens consecutivas fornecessem valores de massa constante. Considerou-se, para efeito de cálculos, que a umidade de equilíbrio foi atingida quando não ocorreu mais variação do peso. A umidade em cada instante foi determinada conforme a Equação 2.
$\mathrm{X}(\mathrm{b} . \mathrm{s})=\frac{\mathrm{M}_{\text {tempo }}-\mathrm{M}_{\text {seca }}}{\mathrm{M}_{\text {seca }}}$

Ao final da secagem, uma parte da amostra foi retirada para determinação da massa de matéria seca, de acordo com a Equação 1.

As curvas de taxa de secagem (N) foram obtidas a partir da derivação das respectivas curvas de secagem pelo método numérico, obtendo-se o valor da umidade nos pontos originalmente utilizados na construção das curvas de secagem, conforme Motta Lima (1999).

Aos dados experimentais de secagem foram ajustados oito modelos matemáticos, que estão apresentados na Tabela 1 , que são frequentemente utilizados para secagem de produtos agrícolas.

As razões de umidade (RU) do produto foram determinadas pela equação 3 .

$\mathrm{RU}=\frac{\mathrm{X}_{\mathrm{t}}-\mathrm{X}_{\mathrm{e}}}{\mathrm{X}_{0}-\mathrm{X}_{\mathrm{e}}}$

Os ajustes dos modelos foram realizados com auxílio do software Statistica $8.0 \circledR$, por meio de análises de regressão não linear, pelo método Quasi-Newton, critério de convergência 0,000099. A avaliação dos modelos foi feita com base nos valores do coeficiente de determinação $\left(\mathrm{R}^{2}\right)$, quiquadrado $\left(\chi^{2}\right)$, raiz do quadrado médio residual (RQMR) e erro médio relativo (PE). As equações de 4 a 6 apresentam como foram calculados os valores do $\chi 2$, RQMR e PE, respectivamente.

$$
\begin{aligned}
& \mathrm{X}^{2}=\frac{\left[\sum_{\mathrm{i}=1}^{\mathrm{N}}\left(\mathrm{RU}_{\exp , \mathrm{i}}-\mathrm{RU}_{\mathrm{pre}, \mathrm{i}}\right)^{2}\right]}{\mathrm{N}-\mathrm{n}} \\
& \mathrm{RQMR}=\left[\frac{\sum_{\mathrm{i}=1}^{\mathrm{N}}\left(\mathrm{RU}_{\mathrm{pre}, \mathrm{i}}-\mathrm{RU}_{\exp , \mathrm{i}}\right)^{2}}{\mathrm{~N}}\right]^{\frac{1}{2}} \\
& \operatorname{PE}(\%)=\frac{100}{\mathrm{~N}}\left(\sum_{\mathrm{i}=1}^{\mathrm{N}} \frac{\left|\mathrm{U}_{\exp , \mathrm{i}}-\mathrm{U}_{\text {pre }, \mathrm{i}}\right|}{\mathrm{U}_{\exp , \mathrm{i}}}\right)
\end{aligned}
$$


Tabela 1 - Modelos matemáticos utilizados para descrever o processo de secagem da semente de moringa.

\begin{tabular}{|c|c|c|}
\hline Nome & Modelo & Referência \\
\hline $\begin{array}{l}\text { Aproximação da } \\
\text { Difusão } \\
\end{array}$ & $R U=a \cdot \exp (-k \cdot t)+(1-a) \cdot \exp (-k \cdot b \cdot t)$ & $\begin{array}{c}\text { Yaldiz, Ertekin e Uzum } \\
(2001)\end{array}$ \\
\hline Dois Termos & $R U=a \cdot \exp \left(-k_{0} \cdot t\right)+b \cdot \exp \left(-k_{1} \cdot t\right)$ & Henderson (1974) \\
\hline Henderson e Pabis & $\mathrm{RU}=\mathrm{a} \cdot \exp (-\mathrm{k} \cdot \mathrm{t})$ & Henderson \& Pabis (1961) \\
\hline $\begin{array}{l}\text { Henderson e Pabis } \\
\text { Modificado } \\
\end{array}$ & $R U=a \cdot \exp (-k \cdot t)+b \cdot \exp \left(-k_{1} \cdot t\right)+c \cdot \exp \left(-k_{2} . t\right)$ & Henderson \& Pabis (1961) \\
\hline Logarítmico & $\mathrm{RU}=\mathrm{a} \cdot \exp (-\mathrm{k} \cdot \mathrm{t})+\mathrm{c}$ & Togrul \& Pehlivan (2004) \\
\hline Page & $\mathrm{RU}=\exp \left(-\mathrm{k} \cdot \mathrm{t}^{\mathrm{n}}\right)$ & Page (1949) \\
\hline Thompson & $R U=\exp \left\{\left[-a-\left(a^{2}+4 \cdot t^{2}\right)^{0,5}\right] / 2 \cdot b\right\}$ & Thompson (1968) \\
\hline Verna & $R U=a \cdot \exp (-k \cdot t)+(1-a) \cdot \exp \left(-k_{1} \cdot t\right)$ & Verna et al. (1985) \\
\hline
\end{tabular}

A escolha dos melhores ajustes, baseouse nos maiores valores do $\mathrm{R}^{2}$ e os menores valores do $\chi 2$ e RQMR. Segundo Madamba, Driscoll e Buckle (1996) um erro médio relativo abaixo de $10 \%$ indica um bom ajuste do modelo.

Para a determinação do coeficiente de difusão efetivo, fez-se o ajuste dos dados experimentais da secagem da semente de moringa ao modelo matemático da difusão líquida para a forma geométrica elíptica, de acordo com a Equação 7. Para tempos longos de secagem, com RU>0,6, considera-se $n=1$ (MADAMBA, DRISCOLL $\mathrm{e}$ BUCKLE, 1996).

$\mathrm{RU}=\frac{8}{\pi^{2}} \sum_{\mathrm{n}=1}^{\infty} \frac{1}{(2 \mathrm{n}-1)^{2}} \exp \left[-\frac{(2 \mathrm{n}-1)^{2} \cdot \pi^{2} \cdot \mathrm{D}_{\mathrm{ef}} \mathrm{t}}{4 \cdot \mathrm{L}^{2}}\right]$

Como a difusividade varia conforme mudam as condições de secagem (temperatura e velocidade do ar), ela não é intrínseca ao material. A relação do aumento do coeficiente de difusão com a elevação da temperatura do ar de secagem foi descrita por meio da Equação de Arrhenius (Equação 8).

$\mathrm{D}_{\mathrm{ef}}=\mathrm{D}_{0^{*}} \exp \left(-\frac{\mathrm{E}_{\mathrm{a}}}{\mathrm{R} \cdot \mathrm{T}}\right)$

\section{RESULTADOS E DISCUSSÕES}

A Figura 3 apresenta as curvas de secagem obtidas para as sementes de moringa, mostrando a influência da temperatura no processo de secagem. Observa-se que as curvas apresentam comportamentos semelhantes para cada temperatura, onde com o aumento da temperatura ocorreu uma diminuição do tempo de secagem, evidenciando a maior velocidade na retirada de água.

Figura 3 - Curvas de secagem da semente de moringa, em função a temperatura do ar de secagem.

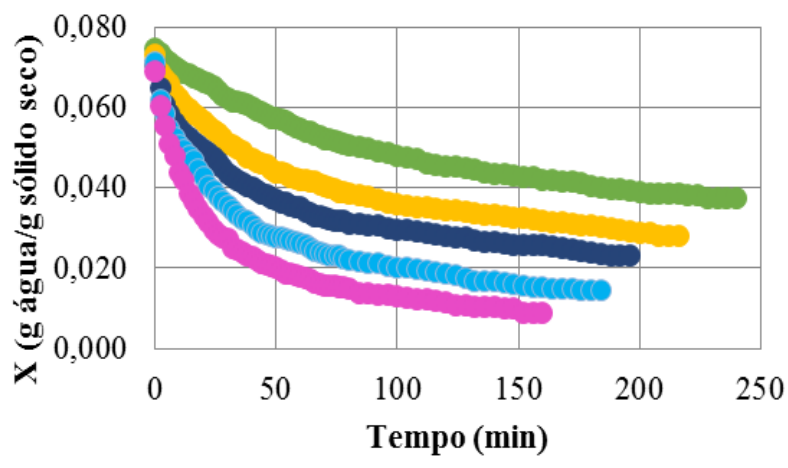

$40^{\circ} \mathrm{C} \cdot 50^{\circ} \mathrm{C} \cdot 60^{\circ} \mathrm{C} \cdot 70^{\circ} \mathrm{C} \cdot 80^{\circ} \mathrm{C}$ 
A Tabela 2 apresenta a umidade equilíbrio da semente de moringa e tempo necessário para atingir esta umidade, de acordo com a temperatura de secagem.

Tabela 2 - Umidade de equilíbrio alcançada para cada temperatura de secagem.

\begin{tabular}{c|c|c|c|c|c}
\hline $\mathbf{T}\left({ }^{\circ} \mathbf{C}\right)$ & $\mathbf{4 0}$ & $\mathbf{5 0}$ & $\mathbf{6 0}$ & $\mathbf{7 0}$ & $\mathbf{8 0}$ \\
\hline $\mathrm{X}_{\mathrm{eq}}$ & 0,037 & 0,028 & 0,023 & 0,014 & 0,009 \\
\hline $\mathrm{t}(\mathrm{min})$ & 228 & 216 & 196 & 184 & 160 \\
\hline
\end{tabular}

Por meio da Figura 3 e Tabela 2 verificou-se que foram obtidas umidades de equilíbrio menores para as maiores temperaturas, isso se deve ao fato de nas maiores temperaturas a umidade relativa do ar ser menor, aumentando a transferência de massa (umidade) da semente para $o$ ar, consequentemente reduzindo o tempo necessário para se atingir o equilíbrio, evidenciando uma maior velocidade de retirada de água.

A Figura 4 apresenta as curvas de taxa de secagem $(\mathrm{N})$ para a avaliação da influência da temperatura no processo de secagem, obtidas a partir da derivação dos dados de umidade (X) em relação ao tempo.

Figura 4 - Curva de taxa de secagem para a semente de moringa, em função a temperatura do ar de secagem.

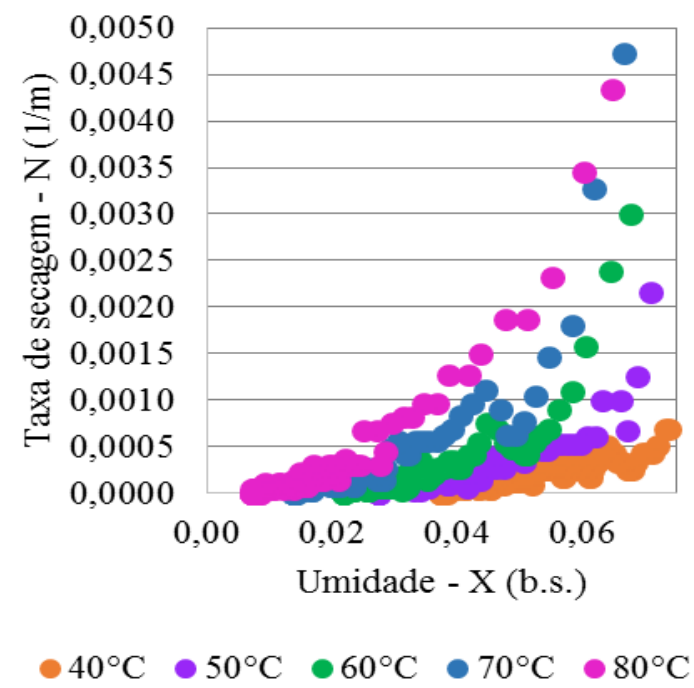

Por meio da Figura 4 verifica-se que ao aumentar a temperatura do ar aumenta-se a velocidade de secagem (taxa de secagem), isso ocorre, pois, ao aumentar a temperatura aumenta-se a transferência de calor para o material, fazendo com que a velocidade de migração da água do interior para a superfície e ambiente seja maior. Também, é evidente que a velocidade de secagem diminui continuamente com o tempo, de acordo com a proximidade da umidade do material com a sua umidade de equilíbrio, representando uma resistência da água na transferência de massa e calor das células internas até a superfície do material.

As curvas não apresentam o período de acondicionamento e nem o de taxa constante, sendo verificado apenas o período de taxa decrescente. Isto significa que a superfície do material não está saturada com água, de acordo com Doymaz e Pala (2003) este fenômeno indica que taxa de secagem é controlada pelo mecanismo de difusão interna. Segundo Ponciano, Driscoll e Buckle (1996) produtos biológicos apresentam este tipo de curva característica.

Os dados obtidos experimentalmente foram ajustados aos modelos matemáticos e os resultados encontram-se dispostos nas Tabelas 8 a 12. De um modo geral, todos os modelos apresentaram bons ajustes, com coeficientes de determinação superiores a 0,97, entretanto de acordo com Madamba, Driscoll e Buckle (1996) apenas o coeficiente de determinação $\left(\mathrm{R}^{2}\right)$ não é capaz de determinar satisfatoriamente $\mathrm{o}$ melhor modelo, tornando-se viável avaliação dos demais parâmetros estatísticos.

Desta forma, os modelos de Aproximação da Difusão e Verna apresentaram bons ajustes para as temperaturas de $50,60,70$ e $80^{\circ} \mathrm{C}$, onde os valores de $\mathrm{R}^{2}$ variaram de 99,74 a $99,94 \%$ e o PE de 14,51 a 5,09\%, em que os valores do erro médio relativo (PE) indicam o desvio dos valores observados em relação à curva 
estimada pelo modelo. As Figuras 5 apresentam os dados experimentais de 50 a $80^{\circ} \mathrm{C}$ ajustados aos modelos.

Figura 5 - Dados experimentais de as temperaturas de 50 a $80^{\circ} \mathrm{C}$ ajustados aos modelos da Aproximação da Difusão e Verna.

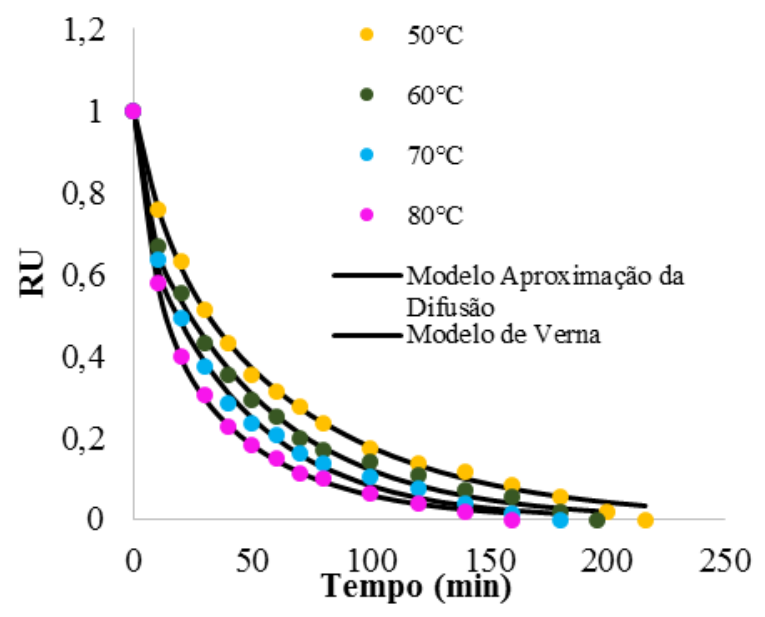

Para os dados experimentais da temperatura de secagem de $40^{\circ} \mathrm{C}$ ocorreu uma exceção, na qual o modelo Logarítmico apresentou melhor ajuste seguido do Aproximação da Difusão. $O$ ajuste para o modelo Logarítimico é apresentado na Figura 6.

Tabela 8 - Análises estatísticas para os modelos ajustados para a temperatura do ar de secagem de 40 e $50^{\circ} \mathrm{C}$.

\begin{tabular}{c|cccc|cccc}
\hline \multirow{2}{*}{ Modelos } & \multicolumn{4}{|c|}{ Temperatura 40 $^{\circ} \mathbf{C}$} & \multicolumn{4}{c}{ Temperatura 50 } \\
& $\mathbf{R}^{\mathbf{2}}$ & $\mathbf{X}^{\mathbf{2}}$ & $\mathbf{R Q M R}$ & $\mathbf{P E}(\boldsymbol{\%})$ & $\mathbf{R}^{\mathbf{2}}$ & $\mathbf{X}^{\mathbf{2}}$ & $\mathbf{R Q M R}$ & $\mathbf{P E}(\boldsymbol{\%})$ \\
\hline Aproximação da Difusão & 0,9983 & 0,00028 & 0,016 & 12,23 & 0,9986 & 0,00019 & 0,0134 & 8,91 \\
Dois Termos & 0,9980 & 0,00034 & 0,018 & 14,89 & 0,9987 & 0,00017 & 0,0126 & 8,99 \\
Henderson e Pabis & 0,9980 & 0,00033 & 0,018 & 14,83 & 0,9957 & 0,00055 & 0,0232 & 12,15 \\
Henderson e Pabis Mod. & 0,9980 & 0,00035 & 0,018 & 14,83 & 0,9989 & 0,00015 & 0,0119 & 9,04 \\
Logarítmico & 0,9994 & 0,00011 & 0,010 & 5,36 & 0,9967 & 0,00042 & 0,0201 & 13,95 \\
Newton & 0,9980 & 0,00033 & 0,018 & 14,69 & 0,9893 & 0,00134 & 0,0364 & 17,77 \\
Page & 0,9982 & 0,00031 & 0,017 & 13,28 & 0,9986 & 0,00017 & 0,0130 & 10,01 \\
Thompson & 0,9980 & 0,00033 & 0,018 & 14,81 & 0,9939 & 0,00078 & 0,0275 & 13,77 \\
Verna & 0,9980 & 0,00034 & 0,018 & 14,84 & 0,9986 & 0,00019 & 0,0134 & 8,91 \\
\hline
\end{tabular}

Figura 6 - Dados experimentais de todas as temperaturas ajustados ao modelo Logarítmico, para a temperatura de $40^{\circ} \mathrm{C}$.

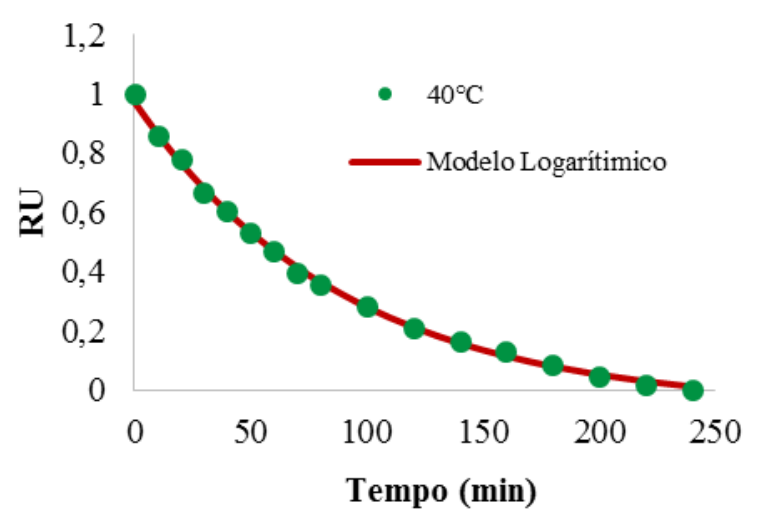

Ressalta-se que o modelo da Aproximação por Difusão foi recomendado para representação da cinética de secagem de alguns produtos, como grãos de crambe (FARIA et al., 2012), entre outros. Silva (2013) observou que o modelo de Handerson e Pabis Modificado foi o que melhor se ajustou aos dados experimentais da secagem de semente de moringa em estufa com circulação de ar forçada. 
Tabela 9 - Análises estatísticas para os modelos ajustados para a temperatura do ar de secagem de 60 e $70^{\circ} \mathrm{C}$.

\begin{tabular}{|c|c|c|c|c|c|c|c|c|}
\hline \multirow{2}{*}{ Modelos } & \multicolumn{4}{|c|}{ Temperatura $40^{\circ} \mathrm{C}$} & \multicolumn{4}{|c|}{ Temperatura $50^{\circ} \mathrm{C}$} \\
\hline & $\mathbf{R}^{2}$ & $\mathbf{X}^{2}$ & RQMR & PE $(\%)$ & $\mathbf{R}^{2}$ & $\mathbf{X}^{2}$ & RQMR & PE $(\%)$ \\
\hline Aproximação da Difusão & 0,9980 & 0,00023 & 0,0148 & 9,70 & 0,9974 & 0,00029 & 0,0165 & 14,51 \\
\hline Dois Termos & 0,9980 & 0,00023 & 0,0148 & 9,66 & 0,9977 & 0,00026 & 0,0155 & 16,92 \\
\hline Henderson e Pabis & 0,9929 & 0,00078 & 0,0276 & 12,96 & 0,9921 & 0,00084 & 0,0286 & 16,21 \\
\hline Henderson e Pabis Mod. & 0,9980 & 0,00024 & 0,0148 & 9,66 & 0,9954 & 0,00027 & 0,0155 & 16,92 \\
\hline Logarítmico & 0,9947 & 0,00060 & 0,0239 & 13,41 & 0,9938 & 0,00067 & 0,0254 & 32,28 \\
\hline Newton & 0,9777 & 0,00241 & 0,0488 & 24,71 & 0,9773 & 0,00237 & 0,0483 & 27,43 \\
\hline Page & 0,9980 & 0,00022 & 0,0148 & 9,77 & 0,9979 & 0,00022 & 0,0146 & 23,70 \\
\hline Thompson & 0,9889 & 0,00122 & 0,0344 & 15,88 & 0,9880 & 0,00128 & 0,0352 & 18,98 \\
\hline Verna & 0,9980 & 0,00023 & 0,0148 & 9,70 & 0,9974 & 0,00029 & 0,0165 & 14,51 \\
\hline
\end{tabular}

Tabela 10 - Análises estatísticas para os modelos ajustados para a temperatura do ar de secagem de $80^{\circ} \mathrm{C}$.

\begin{tabular}{ccccc} 
Modelos & \multicolumn{4}{c}{ Temperatura 80 } \\
& $\mathbf{R}^{\mathbf{2}}$ & $\mathbf{X}^{\mathbf{2}}$ & $\mathbf{R Q M R}$ & PE (\%) \\
\hline Aproximação da Difusão & 0,9994 & 0,00006 & 0,0074 & 5,09 \\
Dois Termos & 0,9995 & 0,00005 & 0,0070 & 5,17 \\
Henderson e Pabis & 0,9888 & 0,00114 & 0,0333 & 23,90 \\
Henderson e Pabis Mod. & 0,9995 & 0,00005 & 0,0070 & 5,17 \\
Logarítmico & 0,9932 & 0,00071 & 0,0259 & 22,63 \\
Newton & 0,9774 & 0,00227 & 0,0472 & 35,56 \\
Page & 0,9991 & 0,00009 & 0,0093 & 7,33 \\
Thompson & 0,9845 & 0,00158 & 0,0391 & 28,46 \\
Verna & 0,9994 & 0,00006 & 0,0074 & 5,09 \\
\hline
\end{tabular}


Os resultados obtidos para o coeficiente de difusividade em cada temperatura de secagem estão dispostos na Figura 7.

Figura 7 - Valores do coeficiente de difusão $\left(\mathrm{m}^{2} / \mathrm{s}\right)$ obtidos para a secagem da semente de moringa nas temperaturas de $40,50,60,70$ e $80^{\circ} \mathrm{C}$.

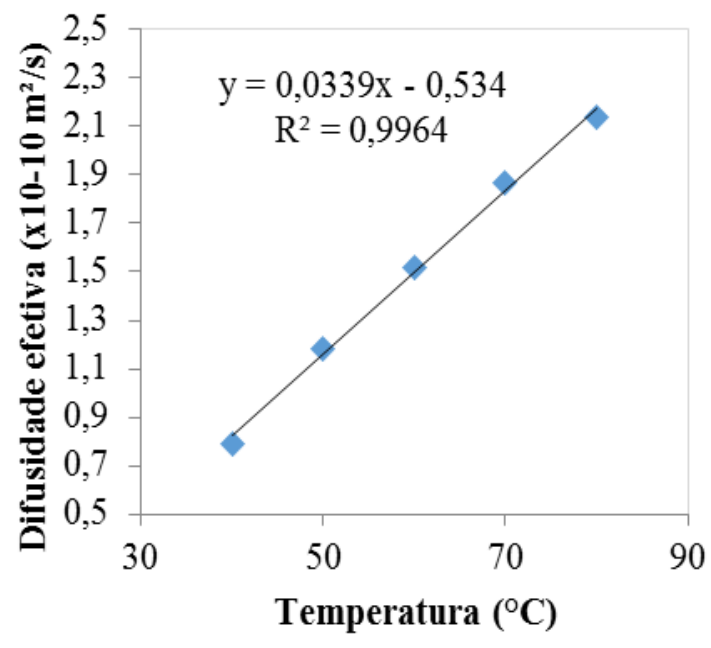

O coeficiente de difusividade não é uma propriedade intrínseca ao material, pois varia com as condições de secagem e características do material. Este fato é observado na Figura 7 , onde é possível verificar que o coeficiente de difusividade aumenta linearmente $\left(\mathrm{R}^{2}=99,64 \%\right)$ com a elevação da temperatura do ar de secagem, ou seja, o aumento da temperatura do ar de secagem facilita $o$ processo de migração da água do interior para a superfície do material. A difusividade da água na semente de moringa variou $0,79 \times 10^{-10}$ a $2,14 \times 10^{-10}$, para as temperaturas de 40 a $80^{\circ} \mathrm{C}$. Segundo Madamba, Driscoll e Buckle (1996) produtos alimentícios apresentam a difusividade efetiva na faixa de $10^{-9}$ a $10^{-11}$ $\mathrm{m}^{2} / \mathrm{s}$

A relação da temperatura do ar de secagem com a difusividade efetiva é representada pela Equação de Arrhenius, conforma mostra a Figura 8.
Figura 8 - Representação de Arrhenius para o coeficiente de difusão, em função da temperatura do ar de secagem da semente de moringa.

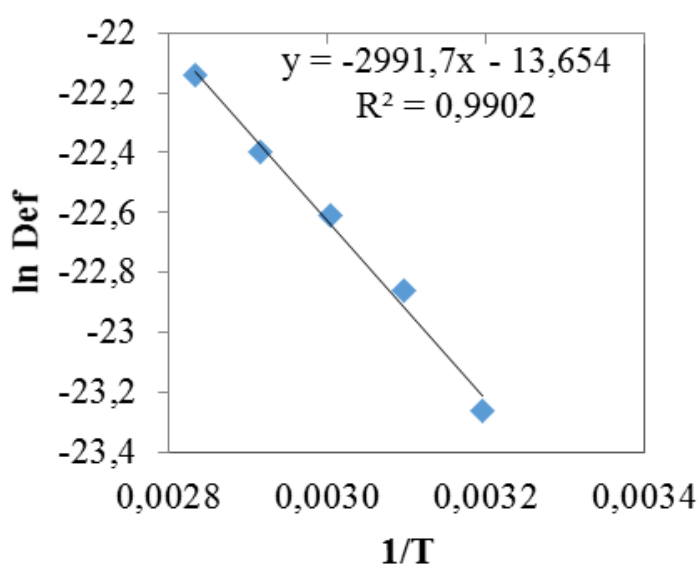

Através do coeficiente angular da reta, apresentado na Figura 8, obtém-se a energia de ativação por meio da Equação 8, onde o valor encontrado foi de $24,33 \mathrm{~kJ} / \mathrm{mol}$. A energia de ativação é facilidade com que as moléculas de água superam a barreira para que o processo de difusão possa ser desencadeado, tal que quanto menor a energia de ativação maior será a difusividade de água no material. Segundo Zogzas, Maroulis e Marinos-Kouris (1996) a energia de ativação de materiais agrícolas varia entre 12,7 a 110 $\mathrm{kJ} / \mathrm{mol}$.

\section{CONCLUSÕES}

Dentre os modelos testados, o da Aproximação por Difusão representa satisfatoriamente o processo de secagem das sementes de moringa em camada fina, para as temperaturas de $50,60,70$ e $80^{\circ} \mathrm{C}$. Para a temperatura de $40^{\circ} \mathrm{C}$ o modelo que melhor se ajustou foi o Logarítimico.

O coeficiente de difusão aumenta com a elevação da temperatura, apresentando valores entre $0,79 \times 10^{-10}$ e $2,15 \times 10^{-10} \mathrm{~m}^{2} / \mathrm{s}$, para uma espessura equivalente de $6,8 \mathrm{~mm}$ e temperaturas entre 40 a $80{ }^{\circ} \mathrm{C}$.

A relação do coeficiente de difusão com a temperatura de secagem pode ser descrita pela equação de Arrhenius, que apresenta 
energia de ativação para a difusão líquida durante a secagem das sementes de moringa, de $24,33 \mathrm{~kJ} / \mathrm{mol}$.

\section{NOMENCLATURA}

$\mathrm{X}$ (b.s.): razão entre a massa de água e a massa de sólido seca em um determinado tempo;

Múmida: massa de semente e água presente na amostra, g;

$\mathrm{M}_{\text {seca }}$ : massa da semente seca obtida em estufa, g.

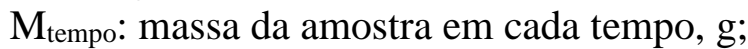

$\mathrm{N}$ : taxa de secagem, $\min ^{-1}$;

RU: razão de umidade;

$\mathrm{k}, \mathrm{k}_{0}, \mathrm{k}_{1}$ : constantes de secagem, $\min ^{-1}$;

a, b, c, n: coeficientes dos modelos;

$\mathrm{t}$ : tempo de secagem, min.

$\mathrm{X}_{\mathrm{t}}$ : umidade em determinado tempo, b.s.;

$\mathrm{X}_{\mathrm{e}}$ : umidade de equilíbrio, b.s.;

$\mathrm{X}_{0}$ : umidade inicial, b.s.;

$\mathrm{RU}_{\text {exp,i: i-ésima razão de umidade }}$

experimental;

$\mathrm{RU}_{\text {pre,i:i-ésima razão de umidade predita pelo }}$

modelo ajustado;

$\mathrm{U}_{\text {exp,i: }}$ i-ésimo teor de água experimental, b.s.;

$\mathrm{U}_{\text {pre,i:i-ésimo teor de água predito pelo }}$

modelo ajustado, b.s.;

$\mathrm{N}$ : número de observações;

n: número de parâmetros do modelo.

$\mathrm{D}_{\text {ef }}$ : coeficiente de difusão efetivo, $\mathrm{m}^{2} / \mathrm{s}$;

L: metade da espessura da amostra, m;

n: número de termos, Equação 7;

t: tempo, s, Equação 7;

L: metade da espessura da amostra, $\mathrm{m}$.

$\mathrm{D}_{0}$ : fator pré-exponecial;

$\mathrm{E}_{\mathrm{a}}$ : energia de ativação, $\mathrm{kJ} / \mathrm{mol}$;

$\mathrm{R}$ : constante universal dos gases, 8,134

$\mathrm{kJ} / \mathrm{kmol} \mathrm{K}$

T: temperatura, K, Equação 8.

\section{REFERÊNCIAS}

AFONSO JÚNIOR, P.C.; CORRÊA, P.C. Comparação de modelos matemáticos para descrição da cinética de secagem em camada fina de sementes de feijão. Revista Brasileira de Engenharia Agrícola e Ambiental, v.3, p.349-353,1999.

ANWAR, F.; BHANGHER, M.I. Analytical Characterization of Moringa oleifera Seed Oil Grown in Temperate Regions of Pakistan. Journal Agricultural and Food Chemistry, v. 51, p. 6558-6563, 2003.

DOYMAZ, I. Drying behaviour of green beans. Journal of Food Engineering, v.66, p. 161-165, 2005.

DOYMAZ, I.; PALA, M. The thin-layer drying characteristics of corn. Journal of Food Engineering, v.60, p. 125-130, 2003.

FARIA, R.Q.; TEIXEIRA, I.R.; DEVILLA, I.A.; ASCHERI, D.P.; RESENDE, O. Cinética de secagem de sementes de crambe. R. Bras. Eng. Agríc. Ambiental, v. 16, n. 5, p. 573-583, 2012.

FRIGHETTO, R.T.S.; FRIGHETTO, N.; SCHNEIDER， R.P.; LIMA， P.C.F. O potencial da espécie Moringa oleifera (Moringaceae).I. A planta como fonte de coagulante natural no saneamento de águas e como suplemento alimentar. Revista Fito, v. 3, n. 2, p. 78-88, 2007.

HENDERSON, S. M. Progress in developing the thin layer drying equation. Transactions of the ASAE, v. 17, p. 1167- 1168, 1974.

HENDERSON, S. M.; PABIS, S., Grain drying theory. I. Temperature effect on drying coefficient. Journal of Agriculture

Engineering, v.33, p. 169-174, 1961.

LUZ, G. R. Modelagem Matemática e Análise do Secador Rotativo de Farelo de Soja. 2006. Dissertação (Mestrado em 
Engenharia Química), Universidade Estadual de Maringá, Maringá, 2006.

MADAMBA, P.S.; DRISCOLL, R.H.; BUCKLE, K.A. The thin-layer drying characteristics of garlic slices. Journal of food engineering, v. 29, n. 1, p. 75-97, 1996

MORTON, J.F. The horseradish tree, Moringa pterygosperma (Moringacea) - A boon to arid lands?. Economic Botany, v. 45, n. 3, p. 318-333, 1991.

MOTTA LIMA, O. C. Estudo sobre a secagem de folhas de celulose. 1999. Tese (Doutorado em Engenharia Química), Universidade Federal do Rio de Janeiro, Rio de Janeiro, 1999.

PAGE, G.E. Factors influencing the maximum rates of air drying shelled corn in thin layers. Indiana, 1949, Thesis (M. Sc.), Purdue University.

PONCIANO, S.M.; DRISCOLL, R.H.; BUCKLE, K.A. The Thin-layer Drying Characteristics of Garlic Slices. Journal of Food Engineering, v. 29, p. 75-97, 1996.

SILVA, M.O. Caracterização física e química da semente de Moringa oleífera e estudo do processo de obtenção de óleo. 2013. 50 f. Dissertação (Mestrado em Engenharia de Alimentos). Departamento de Engenharia de Alimentos, Universidade Estadual de Maringá, Maringá, 2013.

THOMPSON, T. L; PEART, R. M.; FOSTER, G. H. Mathematical simulation of corn drying - A new model. Transaction of the ASAE, v. 11, n. 4, p. 582-586, 1968.

TOĞRUL, İ. T.; PEHLIVAN, D. Modelling of thin layer drying kinetics of some fruits under open-air sun drying process. Journal of
Food Engineering, v. 65, n. 3, p. 413-425, 2004.

VERNA, L. R.; BUCKLIN, R. A.; ENDAN, J. B.; WRATTEN, F. T.Effects of drying air parameters on rice drying models.

Transactions of the ASAE, v. 28, p. 296301, 1985.

YALDIZ, O.; ERTEKIN, C.; UZUN, H. I. Mathematical modeling of thin layer solar drying of sultana grapes. Energy, v. 26, n. 5, p. 457-465, 2001.

ZOGZAS, N. P.; MAROULIS, Z. B.; MARINOS-KOURIS, D. Moisture diffusivity data compilation in foodstuffs. Drying technology, v. 14, n. 10, p. 2225-2253, 1996.

\section{AGRADECIMENTOS}

A CAPES pelo auxílio financeiro. 